\title{
Local mismatch location and spatial scale detection in image Registration
}

\author{
R Narayanan ${ }^{1}$, J A Fessler ${ }^{1,2,3}$, B Ma $^{2}$, H Park $^{2}$ and C R Meyer ${ }^{1,2}$ \\ ${ }^{1}$ Department of Biomedical Engineering, ${ }^{2}$ Department of Radiology and ${ }^{3}$ Department of \\ Electrical Engineering and Computer Science. \\ Ann Arbor, MI, 48109, USA
}

\begin{abstract}
Image registration is now a well understood problem and several techniques using a combination of cost functions, transformation models and optimizers have been reported in medical imaging literature. Parametric methods often rely on the efficient placement of control points in the images, that is, depending on the location and scale at which images are mismatched. Poor choice of parameterization results in deformations not being modeled accurately or over parameterization, where control points may lie in homogeneous regions with low sensitivity to cost. This lowers computational efficiency due to the high complexity of the search space and might also provide transformations that are not physically meaningful, and possibly folded.

Adaptive methods that parameterize based on mismatch in images have been proposed. In such methods, the cost measure must be normalized, heuristics such as how many points to pick, resolution of the grids, choosing gradient thresholds and when to refine scale would have to be ascertained in addition to the limitation of working only at a few discrete scales.

In this paper we identify mismatch by searching the entire image and a wide range of smooth spatial scales. The mismatch vector, containing location and scale of mismatch is computed from peaks in the local joint entropy. Results show that this method can be used to quickly and effectively locate mismatched regions in images where control points can be placed in preference to other regions speeding up registration.
\end{abstract}

Keywords: image, registration, scale, joint, entropy, mismatch, deformation

\section{INTRODUCTION}

Image registration is a widely published and growing area in medical imaging. Several algorithms have been proposed that are capable of recovering large and complex deformations. Using mutual information for image registration ${ }^{1}$ has furthered the capability of these algorithms because of their extensibility to multimodality images. This has also spurred the need for faster methods that can provide similar performance using smaller degrees of freedom eliminating control points in regions where they have little or no effect.(E.g. ${ }^{2,3}$ ) as opposed to a uniform grid placement.

Often an image pair is aligned initially based on an affine transformation before a nonlinear warp is applied. The nonlinear warp may be parametric (e.g., thin plate splines, ${ }^{4} \mathrm{~B}$-splines ${ }^{5}{ }^{5}$ compact radial basis functions ${ }^{6}$ or other parameterizations ${ }^{2,5,7-11}$ ), or non-parameterized free-form deformation fields based on an elastic ${ }^{12,13}$ or viscous fluid flow ${ }^{14,15}$ regularization.

Regardless of the specific deformation model used, the time needed to complete registration is related to the extent of geometrical misalignment, the time taken for each cost computation, and the total number of DOF used to describe the deformation. In certain applications the images may not belong to the same modality, necessitating the use of Mutual Information (MI) based methods ${ }^{1}$ that maybe more time consuming than Sum of Squared Differences (SSD) or correlation. Even for intra-modality registration, MI is often used because of its robustness to differences in scanner parameters. Also registration times tend to be high if the images are large

Further author information: (Send correspondence to R.N.)

R.N.: E-mail: rnz@umich.edu, Telephone: 17346470848.

Funding for this research was provided by DHHS-, PHS-, NIH-, NCI \#1P01CA87634

Medical Imaging 2007: Image Processing, edited by Josien P. W. Pluim, Joseph M. Reinhardt, Proc. of SPIE Vol. 6512, 65121X, (2007) · 1605-7422/07/\$18 - doi: 10.1117/12.707439 
and deformations are complex with the added requirement that user intervention is impractical. However often we can use fewer DOF if we place control points at locations having the most image mismatch.

There have been some papers that discuss spatially preferential parameterizations prior to registration. Rohlfing et al. ${ }^{16}$ used multi-level B-splines for registration where a control point at a particular scale was held fixed (i.e., not optimized) if its local marginal entropies on the target and floating images were below $50 \%$ of the respective maximum entropies. Rohde et al., ${ }^{2,17}$ in their compactly supported radial basis function strategy, used only those control points that produced large gradients of cost. Schnabel et al. ${ }^{18}$ also picked active control points based on cost gradients for their B-spline method. Park et al. ${ }^{3,19}$ added thin plate spline control points at each iteration at locations with large local mismatch. As far as we are aware, thus far no thorough analysis has been made to find mismatched regions and their degree of mismatch through an analysis of a wide range of spatial scales at all locations in the image pair prior to registration. For any of the several deformation models used, the knowledge of location and spatial extent of deformation could help drastically reduce compute times by avoiding parameterization of local regions already well aligned. This enables algorithms to work at several distinct locations at specific scales and yet contain the dimensionality of the optimization search space.

The scale of a deformation model relates to the notion of scale space. Koenderink ${ }^{20}$ discussed three aspects of scale in images: pixellation, i.e., inner scale, extent of the Regions Of Interest (ROI), i.e., outer scale and the resolution of the histogram used to describe the intensity distribution of the image, i.e., tonal scale. In this paper we focus on the spatial extent of the mismatch between images so we will only use outer scale. The estimated mismatch vector contains the location and outer scale at which the images are mismatched. Any reference further to scale in the paper is to outer scale.

The ideas discussed here are extensions to commonly used approaches in the vision community to quantify local complexity in images using information theoretic measures. Gilles ${ }^{21}$ used Shannon entropy to quantify local image complexity. Salient patches identified as entropy peaks in scale space were used to estimate a global transformation between aerial reconnaissance images. Jagersand ${ }^{22}$ used Kullback contrast between successive scales as a measure of differential information gain at a particular scale. Kadir ${ }^{23}$ made several extensions to Gilles' idea notably the search through a wide range of scales in color images containing complex scenes and the use of a new saliency measure that weighs the entropy by a measure of self-dissimilarity in scale space. In this paper we use a similar approach to estimate feature mismatch for a pair of images using joint entropy. This makes it possible to study the mismatch of image pairs through feature space and outer scale simultaneously. Further, we have computed mismatch through continuous outer scale (Gaussian) giving smooth joint entropy estimates. We begin section 2 by defining the joint histogram and joint entropy of image pairs through location and scale. We have included several examples to show that the mismatch vectors are in excellent agreement with location and scale of synthetic deformations.

\section{MISMATCH MEASURE}

We propose to locate feature mismatch for a pair of images using joint entropy that is computed from local histograms. In three dimensions each mismatch vector found is made of individual location components $C_{x}, C_{y}$ and $C_{z}$, and scale component $\alpha$ corresponding to mismatch and is of the form $\left[\begin{array}{lllll}C_{x} & C_{y} & C_{z} & \alpha\end{array}\right]^{T}$, or $\left[\begin{array}{lll}C_{x} & C_{y} & \alpha\end{array}\right]^{T}$ in two dimensions.

\subsection{Local Histogram}

The local joint histogram for a pair of images captures the distribution of intensities within a locally specified support. Koenderink ${ }^{20}$ used histograms to represent local information content in images parameterized based on the "inner scale" (gaussian blurring window), "outer scale" (gaussian region of interest) and histogram resolution. We define the local joint histogram of two images, $I_{1}(\mathbf{r})$ and $I_{2}(\mathbf{r})$ as

$$
h\left(i_{1}, i_{2} ; \alpha, \beta, \mathbf{r}_{0}\right)=\frac{1}{2 \pi \alpha^{2}} \sum_{\mathbf{r}} A\left(\mathbf{r} ; \mathbf{r}_{0}, \alpha\right) e^{-\frac{\left(I_{1}(\mathbf{r}, \sigma)-i_{1}\right)^{2}}{2 \beta^{2}}} e^{-\frac{\left(I_{2}(\mathbf{r}, \sigma)-i_{2}\right)^{2}}{2 \beta^{2}}}
$$

where $A\left(\mathbf{r} ; \mathbf{r}_{0}, \alpha\right)=e^{\frac{-\left(\mathbf{r}-\mathbf{r}_{0}\right)^{2}}{2 \alpha^{2}}}$ denotes a gaussian window function with "outer scale" parameter $\alpha$ centered at $\mathbf{r}_{0}$, and $I_{k}(\mathbf{r}, \sigma)=I_{k}(\mathbf{r}) \otimes G(\mathbf{r}, \sigma)$ where $k=1,2 . i_{1}$ and $i_{2}$ are variables that span the respective image intensities, 

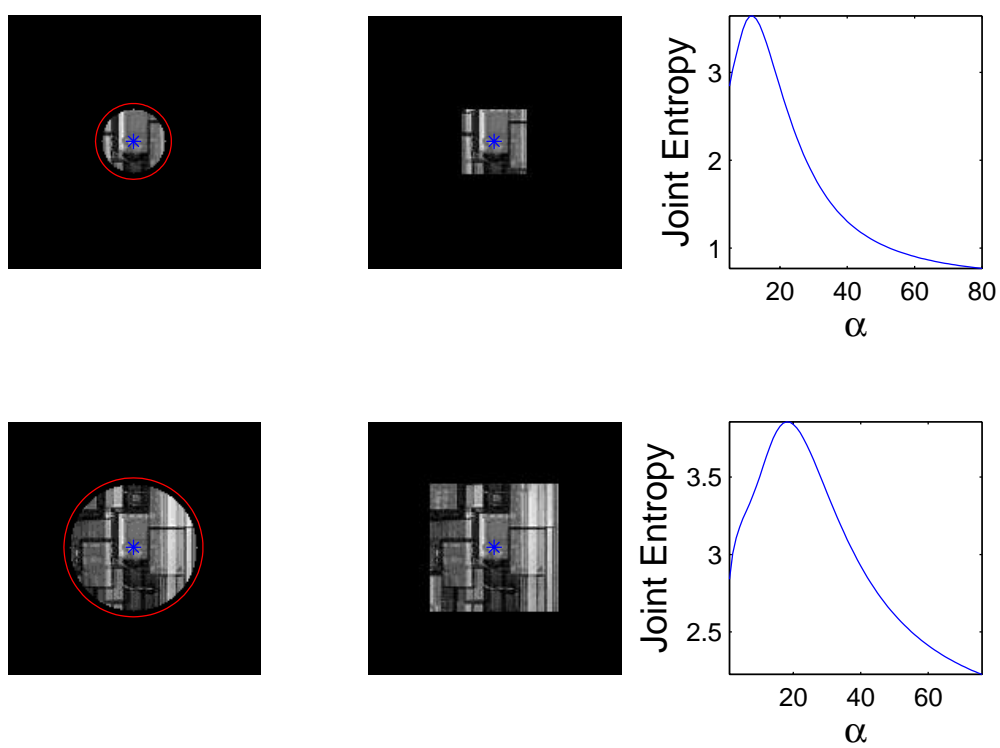

Figure 1. Entropy profile in outer scale. Left column: target images; Middle column: floating images; Right column: joint entropy vs. outer scale for center pixel denoted by ${ }^{*}$, i.e., $H\left(\alpha ; \mathbf{r}_{\text {center }}\right)$.

$G$ is the gaussian kernel of the scale space filter with kernel width $\sigma$, and $\beta$ is the Parzen window width. We are interested in finding the location $\left(\mathbf{r}_{0}\right)$ and region of interest $(\alpha$, i.e. outer scale) over which the two images are misaligned. The $2 \pi \alpha^{2}$ factor is the normalization applied to the spatial gaussian window so that the histogram sums to one. The gaussian window parameterized by $\alpha$ prevents abrupt truncation of features and gives much smoother entropy profiles across scale compared to using a circular window.

\subsection{Local joint entropy}

The joint entropy is measured from the normalized local histogram described above and is computed as

$$
H\left(\alpha ; \mathbf{r}_{0}\right)=-\frac{1}{N_{0}} \sum_{i_{1}} \sum_{i_{2}} h\left(i_{1}, i_{2} ; \alpha, \beta, \mathbf{r}_{0}\right) \log _{2}\left(\frac{h\left(i_{1}, i_{2} ; \alpha, \beta, \mathbf{r}_{0}\right)}{N_{0}}\right)
$$

where $N_{0}$ is the sum of the histogram $h$. ( $N_{0}$ is always 1 except near image boundaries). The spatial scale $(\alpha)$ at which the target image is misaligned with the floating image at location $\mathbf{r}_{0}$ is then estimated over a range of scales using joint entropy. If the two images are locally mis-registered at $\mathbf{r}_{0}$ and scale $\alpha^{*}$, then we expect a peak in joint entropy at that scale.

\subsection{Example1: Sensitivity to scale}

Fig(1) shows the target images (left column) and corresponding floating images (middle column). The images have the same intensities within the shapes (circle and square). Clearly the images in the top row are mismatched at a lower scale in the local region centered at the circle compared to the images in the bottom row. The right column shows their entropy vs outer scale $(\alpha)$. The peaks show the scale at which the corresponding images are mismatched. Circles are drawn on the images in the left column with radius $\alpha^{*}$ that correspond to peaks in joint entropy. Observe that this mismatch in scale can be distinguished easily in outer scale. This is discussed in greater detail in following sections. Additionally the magnitude of entropy at this scale gives us a quantitative estimate of the relative importance of this location and scale. Later in this paper we show examples that demonstrate this and our ability to pick highly deformed regions for placing control points. The joint entropy is computed for the image pair at all locations through a broad range of scales and only peaks in joint entropy centered at each pixel through outer scale are picked as being mis-aligned. It is possible that multiple peaks may be found corresponding to more than one spatial scale being significant at a pixel location. In these cases we choose the scale corresponding to the larger magnitude of entropy. 


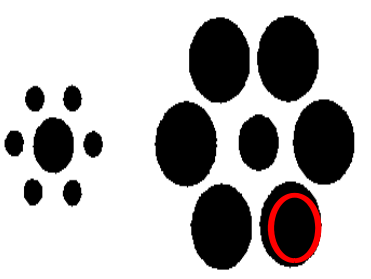

(a)

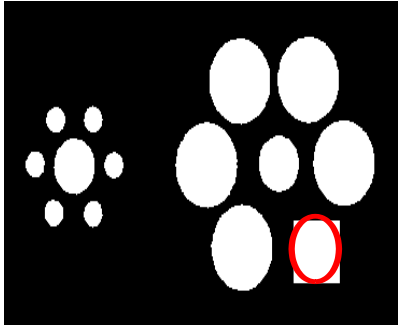

(b)

Figure 2. Search through feature space and scale space a) Target Image, b) Floating image. Center and radius of the red circle shows location and scale of mismatch.

\subsection{Example2: Largest joint entropy location and scale}

Fig. (2) shows several ellipses (2a) and a very similar pattern in (2b) except for the square in one position replacing the ellipse and the inversion of colors. We computed joint entropy over the entire image and found the peaks in scale at every pixel location. The red circle plotted on the first image shows the center of the region and the scale (circle radius) at which the joint entropy was highest in the entire image. Observe that it tracks the boundary of the ellipse in (2a) closely.

\subsection{Example3: Spatial variation of joint entropy}

Fig. (3) shows a local deformation applied to (a) to get image (b). The applied deformation error norm is shown in (c) and (d) is the absolute difference image ((b) and (a)). All calculations are made only along the horizontal line superimposed on the figures to get an idea of the linear spatial variation of peaks and corresponding joint entropy. (e) shows the computed scale at which peaks were found on this line and (f) shows the joint entropy computed at the peaks. The entropy is clearly maximum near the region of the deformation and decreasing with increasing distance from mismatch. The scale that corresponds to the maximum entropy is estimated from (e) which looks bowl shaped because different points on the line find mismatch at different scales. If no peaks were found over the discrete range of scales searched, the scale estimated was set to zero and entropy was not computed (seen as zero in the plot). While points near the deformation estimate scale correctly, distant points find peaks at higher scales. This is dealt with by varying scale of a gaussian lowpass filter as spatial scale increases (discussed in the next subsection). Additionally this also enables us to discriminate local deformations better as will be seen in the next example. The computed scale location and its magnitude are plotted in red (width of two standard deviations of outer scale peak estimated) in (g) and (h) over the intensity difference plot and error norm plot respectively showing how well the estimated location (center of the red line) and scale correspond with the existing mismatch.

\subsection{Inner and Outer scale}

Eq.(1) contains three components of scale. While the Parzen window width $\beta$ is fixed at all spatial scales $(\alpha)$, the degree of smoothing $(\sigma)$ should be related to the spatial scale $(\alpha)$. Jagersand ${ }^{22}$ used different patch sizes at different resolution. In our simulations we varied the scale $\sigma$ with $\alpha$ as $\sigma=\alpha / 3$ i.e., when we are looking at larger regions of interest $(\alpha)$ we are also interested in the variation of coarser features (higher $\sigma$ ).

\subsubsection{Example4: Mismatch location and scale identification}

The importance of varying $\sigma$ with $\alpha$ is shown in Fig. (4) for an image of size $213 \times 291$. (a) is the original image, (b) the deformed image obtained by warping (a) using B-splines, (c) pixel-wise deformation error norm, and (d) shows the positions where peaks in joint entropy were found, where its magnitude corresponds to the scale of deformation. (e) and (f) are respectively the magnitudes of joint entropy obtained by keeping $\sigma$ fixed, and varying $\sigma$ with $\alpha$. In both cases the scale is found to be $\alpha \approx 25$ at the pixel with highest entropy but (f) tracks the deformation error(c) much more closely than (e). The range of scales searched was limited from 6 to 40 , i.e., it was assumed that the maximum scale of the deformation was below 40 . 


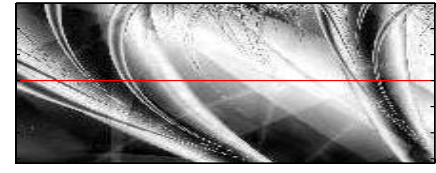

(a)

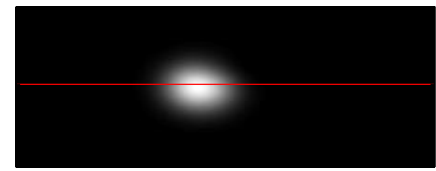

(c)

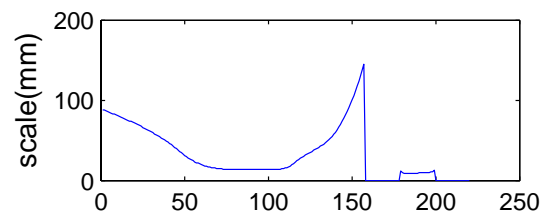

(e)

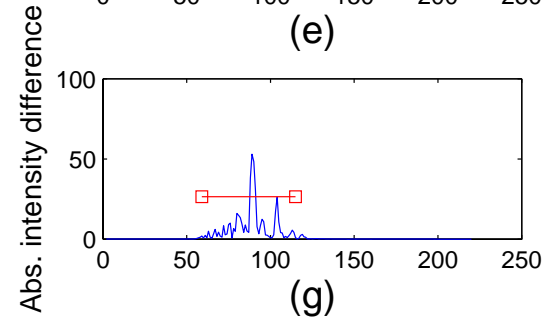

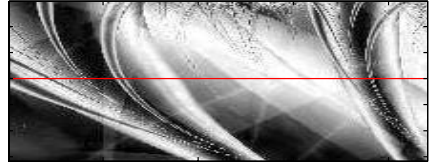

(b)

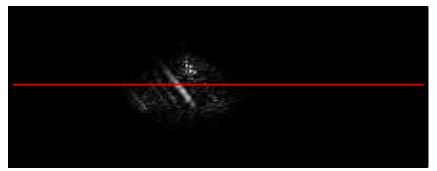

(d)

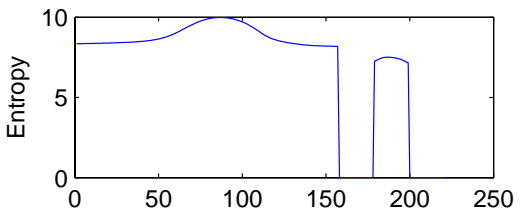

(f)

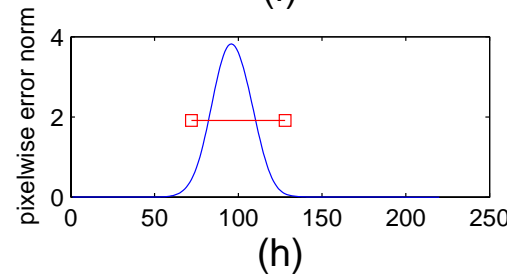

Figure 3. a) Target image, b) Floating image , c) Registration error norm, d) Absolute difference between images in a) and b), e) Significant spatial scale peaks, f) Joint entropy at scale peaks, g) Outer scale found(line)on intensity difference along line profile and h) Outer scale(line) on geometric error norm

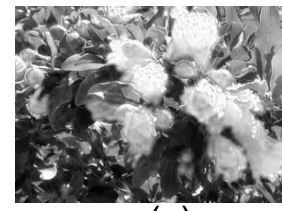

(a)

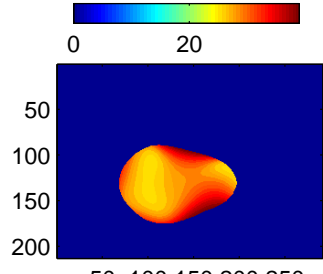

(d)

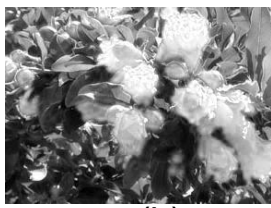

(b)

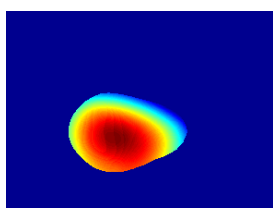

(e)

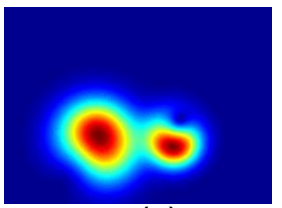

(c)

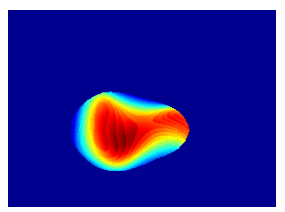

(f)

Figure 4. a) Target image, b) Floating image, c) geometric error norm, d) peaks in scale, e) joint entropy at peaks without gaussian scale space low pass filter and f) joint entropy at peaks with gaussian scale space filter 


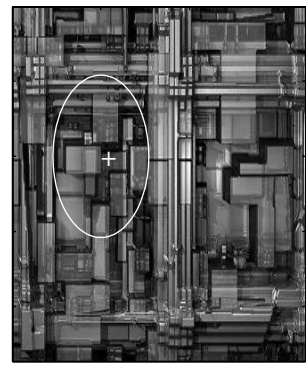

(a)

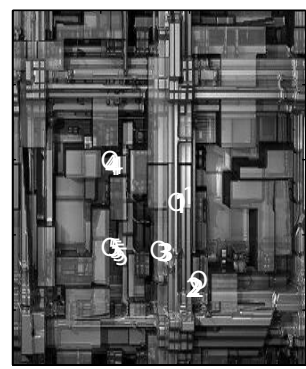

(d)

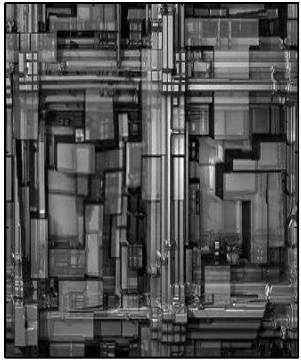

(b)

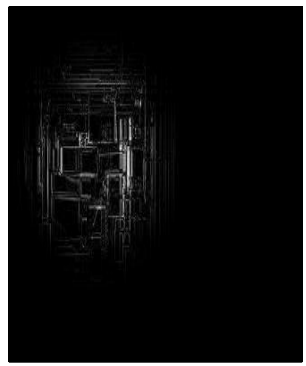

(c)

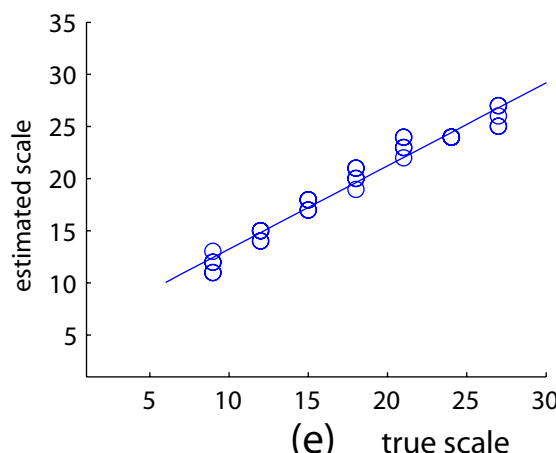

(e) true scale

Figure 5. a) Typical target Image with deformation center and scale, b) Warped floating image, c) Absolute intensity difference, d) Clusters of identified centers, f) Estimated vs. true deformation scale

\section{PRECISION AND ACCURACY OF LOCATION AND SCALE}

In the next experiment, we deformed an image (dimensions $236 \times 330$ ) using a locally affine geometric deforma$\operatorname{tion}^{10}$ at five different locations and four different scales. We applied each deformation independently (20 cases, one location and scale at a time) and the computed the mismatch vector. A typical deformation is shown in Fig. (5a) centered at ' + ' with a deformation spread width ${ }^{10}=27$ pixel units. The circle drawn has a radius equal to twice this width. The corresponding warped image is Fig. (5b) and the absolute intensity difference before and after warp is shown in Fig. (5c).

The circles in Fig. (5d) show the original location of the deformation center and the numbers show the estimated centers for each location. Note that the five numbered markings each are well clustered. Accuracy can be seen visually by the proximity of the markings to the circles. However it possible that regions that are maximally mismatched in intensity may not lie exactly at circle locations, i.e, locations of maximum geometric deformation error norms may not coincide with maximum intensity mismatch so accuracy measurement quantitatively is not possible here but the combined vector precision was found to be small (1.71 pixels) and was computed as the mean deviation of estimated locations from their respective cluster mean vector. Fig. (5e) compares the estimated outer scale with the true scale(kernel width) of the deformation. The scale estimation error was found to be 0.87 pixel units. 


\section{CONCLUSION}

We have shown that mismatched regions in image pairs can be identified by their position and scale based on peaks in joint entropy through a range of outer scales computed at every pixel location. This can be used to parameterize the deformation model for registration, e.g., control point position and support can be initialized for a compact radial basis function. An initialization step of this sort followed by registration may be performed iteratively until no further mismatch is detected or improvement in cost is negligible.

\section{REFERENCES}

1. P. Viola and W. M. Wells, "Alignment by maximization of mutual information," Int. J. Comput. Vision 24(2), pp. 137-154, 1997.

2. G. K. Rohde, A. Aldroubi, and B. M. Dawant, "The adaptive bases algorithm for intensity based nonrigid image registration.," IEEE Trans. Med. Imaging 22(11), pp. 1470-1479, 2003.

3. H. Park, P. H. Bland, K. K. Brock, and C. R. Meyer, "Adaptive registration using local information measures," Medical Image Analysis 8(4), pp. 465-473, 2004.

4. C. R. Meyer, J. L. Boes, B. Kim, P. H. Bland, K. R. Zasadny, P. V. Kison, K. Koral, K. A. Frey, and R. L. Wahl, "Demonstration of accuracy and clinical versatility of mutual information for automatic multimodality image fusion using affine and thin-plate spline warped geometric deformations," Med. Image. Anal. 1, pp. 195-206, 1997.

5. Y. Kybic and M. Unser, "Fast parametric elastic image registration," IEEE Trans. Medical Imaging 12(11), 2003.

6. M. Fornefett, K. Rohr, and H. S. Stiehl, "Radial basis functions with compact support for elastic registration of medical images," in WBIR'99, pp. 173-185, 1999.

7. V. Arsigny, X. Pennec, and N. Ayache, "Polyrigid and polyaffine transformations: A new class of diffeomorphisms for locally rigid or affine registration.," in MICCAI (2), pp. 829-837, 2003.

8. D. Rueckert, L. I. Sonoda, C. Hayes, D. L. G. Hill, M. O. Leach, and D. J. Hawkes, "Nonrigid registration using free-form deformations: Application to breast MR images," IEEE Trans. Med. Imaging 18(8), pp. 712$721,1999$.

9. S. Marsland and C. J. Twining, "Constructing diffeomorphic representations for the groupwise analysis of nonrigid registrations of medical images," IEEE Trans. Medical Imaging 23(8), 2004.

10. R. Narayanan, J. A. Fessler, H. Park, and C. R. Meyer, "Diffeomorphic nonlinear transformations: A local parametric approach for image registration," in IPMI, pp. 174-185, 2005.

11. X. P. Vincent Arsigny, Olivier Commowick and N. Ayache, "A log-euclidean polyaffine framework for locally rigid or affine registration," in WBIR'06, pp. 120-127, 2006.

12. C. Chefd'Hotel, G. Hermosillo, and O. Faugeras, "A variational approach to multi-modal image matching," in VLSM '01: Proceedings of the IEEE Workshop on Variational and Level Set Methods (VLSM'01), p. 21, IEEE Computer Society, (Washington, DC, USA), 2001.

13. R. Bajcsy and S. Kovacic, "Multiresolution elastic matching," Computer vision, graphics and Image processing 46, pp. 1-21, 1989.

14. G. E. Christensen, R. D. Rabbitt, and M. I. Miller, "Deformable templates using large deformation kinematics," Image Processing, IEEE Transactions on 5(10), pp. 1435-1447, 1996.

15. E. D'Agostino, F. Maes, D. Vandermeulen, and P. Suetens, "A viscous fluid model for multimodal non-rigid image registration using mutual information," in MICCAI '02: Proceedings of the 5th International Conference on Medical Image Computing and Computer-Assisted Intervention-Part II, pp. 541-548, SpringerVerlag, (London, UK), 2002.

16. T. Rohlfing and C. R. M. Jr., "Intensity-based non-rigid registration using adaptive multilevel free-form deformation with an incompressibility constraint.," in MICCAI, pp. 111-119, 2001.

17. G. K. Rohde, A. Aldroubi, and B. M. Dawant, "Adaptive free-form deformation for interpatient medical image registration," Medical Imaging 2001: Image Processing 4322(1), pp. 1578-1587, SPIE, 2001. 
18. J. A. Schnabel, D. Rueckert, M. Quist, J. M. Blackall, A. D. Castellano-Smith, T. Hartkens, G. P. Penney, W. A. Hall, H. Liu, C. L. Truwit, F. A. Gerritsen, D. L. G. Hill, and D. J. Hawkes, "A generic framework for non-rigid registration based on non-uniform multi-level free-form deformations," in MICCAI '01: Proceedings of the 4th International Conference on Medical Image Computing and Computer-Assisted Intervention, pp. 573-581, Springer-Verlag, (London, UK), 2001.

19. H. Park and C. Meyer, "Grid refinement in adaptive Non-Rigid registration," in MICCAI, pp. 796-803, 2003.

20. J. J. Koenderink and A. J. V. Doorn, "The structure of locally orderless images," International Journal of Computer Vision 31(2/3), pp. 159-168, 1999.

21. S. Gilles, "Robust description and matching of images," Ph.D. thesis, University of Oxford. , 1998.

22. M. Jagersand, "Saliency maps and attention selection in scale and spatial coordinates: An information theoretic approach,"

23. T. Kadir and M. Brady, "Saliency, scale and image description," International Journal of Computer Vision 45(2), pp. 83-105, 2001. 